\title{
The RANKL/RANK system as a therapeutic target for bone invasion by oral squamous cell carcinoma (Review)
}

\author{
EIJIRO JIMI ${ }^{1,2}$, MASASHI SHIN ${ }^{3}$, HIROYUKI FURUTA ${ }^{1}$, YUKIYO TADA $^{1}$ and JINGO KUSUKAWA $^{4}$ \\ ${ }^{1}$ Division of Molecular Signaling and Biochemistry, Department of Health Improvement, \\ ${ }^{2}$ Center for Oral Biological Research, Kyushu Dental University, Kitakyushu, Fukuoka 803-8580, Japan; \\ ${ }^{3}$ Department of Developmental Biology, Harvard School of Dental Medicine, Cambridge, MA 02142, USA; \\ ${ }^{4}$ Dental and Oral Medical Center, Kurume University School of Medicine, Kurume, Fukuoka 830-0011, Japan
}

Received September 28, 2012; Accepted November 16, 2012

DOI: $10.3892 /$ ijo.2013.1794

\begin{abstract}
Squamous cell carcinomas (SCCs) of the gingiva frequently invade the mandible or maxilla; this invasion is associated with a worse prognosis. The bone destruction associated with carcinomal invasion is mediated by osteoclasts rather than directly by the carcinoma. Therefore, if the cellular and molecular mechanisms by which oral SCC regulates bone invasion were known, it could inform the development of new therapeutic targets. Recently, dysregulation of the functional equilibrium in the receptor activator of $\mathrm{NF}-\kappa \mathrm{B}$ ligand (RANKL)/RANK/osteoprotegerin (OPG) triad has been shown to be responsible for osteolysis associated with the development of malignant tumors in bone sites. Furthermore, the administration of OPG or soluble RANK prevents bone metastasis by cancer cells. In this review, we discuss recent findings indicating that bone invasion by oral SCC is mediated via RANKL/RANK and may be successfully prevented by RANKL inhibition.
\end{abstract}

\section{Contents}

1. Introduction

2. RANKL/RANK system

3. RANKL/RANK signaling is involved in oral SCC cellinduced osteoclastogenesis

4. Osteoclast function regulated by RANKL/RANK signaling

5. The role of RANKL/RANK signaling on bone invasion by oral SCCs

6. Conclusions and perspectives

Correspondence to: Dr Eijiro Jimi, Division of Molecular Signaling and Biochemistry, Department of Health Improvement, Kyushu Dental University, 2-6-1 Manazuru, Kokurakita-ku, Kitakyushu, Fukuoka 803-8580, Japan

E-mail: ejimi@kyu-dent.ac.jp

Key words: oral squamous cell carcinomas, bone invasion, receptor activator of NF- $\mathrm{KB}$ ligand, osteoprotegerin

\section{Introduction}

Malignant tumors of the oral cavity, $95 \%$ of which are squamous cell carcinoma (SCCs), account for approximately $30 \%$ of all head and neck cancers (1). Although oral SCC represents $1-2 \%$ of all human malignancies in Japan, it is the sixth most common cancer worldwide, with more than 500,000 new cases diagnosed each year. The most common sites for SCCs are the tongue and gingiva (1-3).

Carcinoma of the mandibular gingiva, in particular, is associated with bone invasiveness in many patients (Fig. 1A and B) (4-6). Gingival SCC may eventually directly invade the mandible, a feature associated with a worse prognosis. The presence of mandibular invasion is an important criterion for deciding whether surgical intervention is necessary (7). According to the American Joint Committee on Cancer Classification, mandibular invasion is the most advanced primary stage (T4) and overall stage (IV) for these tumors. The 5 -year survival of patients with stage IV oral lesions has been demonstrated to be $39 \%$, as compared with 53,68 and $70 \%$ for stages III, II and I, respectively (8).

The invasion of bone by oral SCCs may be associated with an increase in both osteoclastic and osteoblastic activity. Before invasion of the mandible, SCCs in close proximity to bone initially induce deposition of new bone, especially on the periosteal surface. Two distinct types of invasion of the mandible by oral SCCs have been described $(6,8,9)$. In the erosive pattern the tumor advances on a broad front separated from bone by a layer of connective tissue and osteoclasts are present in the region between bone and stromal tissue. In contrast, the infiltrative pattern of bone invasion is associated with finger and islands of tumor tissue, which invades cancellous spaces with higher osteoclastic activity (Fig. 1C) (10,11). Cases exhibiting features of both patterns are designated as having a mixed pattern. The infiltrative type showed significantly higher rates of positive bone margin and primary site recurrence than the erosive types. In fact, the 3-year disease-free survival of patients with infiltrative and erosive patterns was 30 and $73 \%$, respectively (12). Since these clinicopathological studies indicate that bone invasion by gingival SCC is a critical event that determines prognosis, it is important to develop a therapeutic approach to prevent the bone invasion process. 

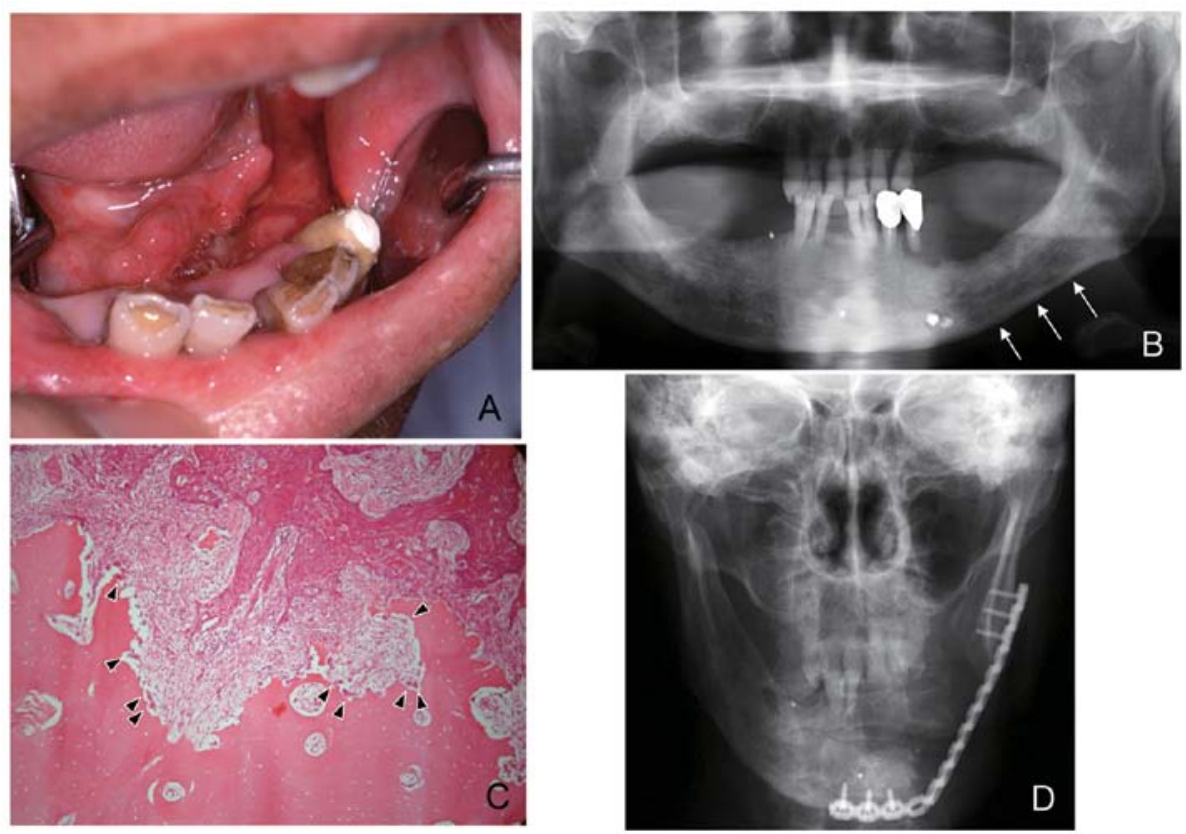

Figure 1. Clinical features of bone invasion by gingival SCCs. (A) Intraoral view of gingival SCCs. (B) Radiographic evidence of bone invasion of the mandible. Arrows outline the lesion. (C) Surgically resected samples were decalcified and stained with Hematoxylin and eosin (H\&E). The infiltrative pattern of bone invasion is characterized by nests and projections of tumor cells along an irregular front, residual bone islands within the tumor, and Haversian system penetration (x100). Arrowheads indicate osteoclasts. (D) Postoperative panoramic radiograph of a patient showing the resected mandible with a free fibula microvascular transfer in place.

Mandibulectomy, if necessary, has a major influence on the patient's quality of life (QOL) and is a critical determinant of postoperative functional outcome (Fig. 1D). Patients with advanced oral SCC have a high mortality, but treatment is complicated by the disruption of speech and swallowing after surgical resection (6-11). It is generally agreed that patients with mandibular invasion should be treated surgically, although the extent of mandibular resection required remains controversial. Resection of the mandibular bone leads to physical damage and frequently also to psychological problems for patients (6-11).

The ability of oral SCCs to invade the maxilla or mandibular bone is a critical factor, which, because it leads to metastasis, affects the prognosis of patients (Fig. 1C) (3). Although controversial, bone destruction that occurs with oral SCC invasion is thought to be mediated by osteoclasts rather than by the carcinoma itself (5). Recent studies have established that bone resorption by osteoclasts is an important step in the process of bone invasion and metastasis in several types of malignancy, indicating that a full understanding of the regulation of osteoclastogenesis by oral SCC cells is necessary to prevent bone invasion by oral SCC cells. Several in vitro and animal experiments using human OSCC cells have shown that tumor cells produce prostaglandins and several cytokines, including interleukin-6 (IL-6), IL-11, TNF $\alpha$ and parathyroid hormone-related protein (PTHrP) $(9,13)$. Indeed, bone is also a storehouse for a variety of cytokines and growth factors and thus provides an extremely fertile environment for cell growth once cancer cells arrest there (Fig. 2) (13). Shibahara et al have reported that the invasive tumors showed a high level of expression of IL-6, IL-11, TNF $\alpha$ and PTHrP (14). In contrast, expression of TGF- $\beta$, IL- $1 \alpha / \beta$ and IL-18 were not different between invasive and non-invasive tumors, suggesting that various cancer-derived cytokines, such as IL-6, IL-11, TNF $\alpha$ and PTHrP, play important roles in bone invasion by oral SCC (14).

Three proteins crucial for osteoclast development and activation are the receptor activator of NF- $\mathrm{BB}$ ligand (RANKL), its receptor, RANK and its decoy receptor, osteoprotegerin (OPG) (15-19). In vitro, the RANKL/RANK signaling pathway, together with macrophage colony-stimulation factor (M-CSF), regulates osteoclast differentiation from monocyte/macrophage progenitors $(15,16)$, whereas the addition of OPG into these culture systems prevents osteoclastogenesis $(17,18)$. Furthermore, these proteins are known to be involved in both normal and pathological bone metabolism. IL-6, IL-11, TNFa and PTHrP released from oral SCCs also induce RANKL expression in osteoblasts or bone marrow stromal cells (19). Thus, RANKL levels are increased in osteolytic lesions associated with malignant tumors, whereas OPG levels are increased in osteoblastic lesions (20). This suggests that blocking RANKL/RANK with soluble RANK (sRANK) or OPG inhibits osteoclastogenesis and successfully prevents bone destruction by cancers (21-23). Therefore, in this review, we first summarize the RANKL/RANK signaling pathway and its effects on osteoclastogenesis. We also describe recent discoveries on the role of RANKL/RANK in bone invasion by oral SCC, based on recent findings from our lab and others. Finally, we discuss the possibility that RANKL inhibition might successfully prevent bone invasion by oral SCC.

\section{RANKL/RANK system}

In the past decade, great progress has been made in understanding bone biology and, in particular, the molecular 


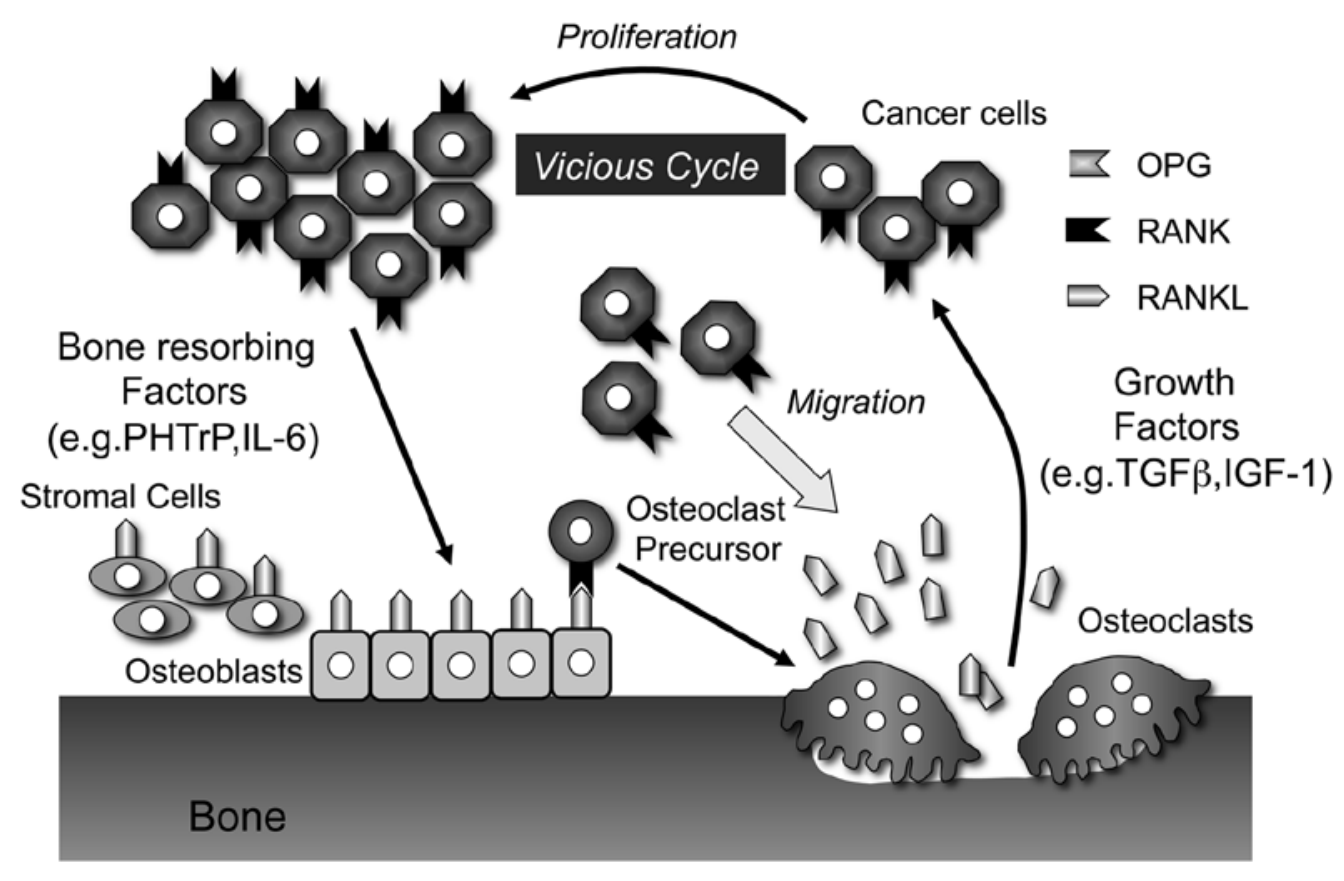

Figure 2. The vicious cycle accelerates bone invasion and cancer cell growth. Bone is a storehouse for various growth factors including insulin-like growth factor and transforming growth factor $\beta$. As a consequence of osteoclastic bone resorption, these growth factors are released into the bone microenvironment in active forms where they stimulate cancer cell proliferation. RANKL released from bone by osteoclasts also induces migration of RANK-expressing SCC cells. The bone resorbtion factors, such as IL-6 and PTHrP, produced by oral SCC cells induce fibroblast stromal cells/osteoblasts to synthesize RANKL or suppress OPG expression. This subsequently induces osteoclast formation and bone resorption activity.

mechanisms of osteoclast development. Osteoclasts, which are present only in bone, are large, multinucleate cells with the capacity to resorb mineralized tissue $(19,24,25)$. Osteoclastic bone resorption consists of multiple steps: the proliferation of osteoclast precursors belong to hematopoietic cells, differentiation of progenitors into mononuclear preosteoclasts (pOCs), fusion of pOCs into multinucleate osteoclasts, clear zone (actin ring) and ruffled border formation (activation), and apoptosis. It has been proposed that osteoblasts or bone marrow stromal cells are involved in osteoclastogenesis through a mechanism involving cell-to-cell contact with osteoclast precursors. This hypothesis was proven by the discovery of RANKL, a member of TNF ligand family $(19,24,25)$.

In 1997, Simonet et al reported the discovery of OPG, an inhibitor of bone resorption (18). OPG is a member of the TNF receptor family but, unlike all other members of the family, lacks a transmembrane domain and represents a secreted TNF receptor (Fig. 3). Tsuda et al independently isolated the same protein as 'osteoclast inhibitory factor (OCIF)', a heparin-binding molecule from the conditioned media of human fibroblast cultures, and showed that its cDNA sequence is identical to that of OPG (17). OPG strongly inhibits osteoclast formation in cocultures of mouse bone marrow cells and primary osteoblasts (POBs) induced by osteotropic factors such as $1 \alpha$ dihydroxyvitamin $\mathrm{D}_{3}$ or $\mathrm{PGE}_{2}$. The administration of OPG to ovariectomized rats causes an increase in bone volume and mineral density associated with a decreased number of osteoclasts (18). Furthermore, OPG-deficient mice exhibit severe osteoporosis caused by enhanced osteoclast formation, suggesting that OPG is a physiological regulator of osteoclast development (26).
A recombinant soluble form of RANKL, together with M-CSF, induces osteoclastogenesis from mouse bone marrow cells or spleen cells in the absence of osteoblasts $(15,16)$. RANKL was first cloned during a search for apoptosis regulatory genes in mouse $\mathrm{T}$ cell hybridoma and was named TNF-related activation-induced cytokine (TRANCE) (27). Two other groups also isolated ligands of OCIF and OPG, respectively, which turned out to be identical to TNF-related activation-induced cytokine (TRANCE) $(15,16)$. RANKL is a type II transmembrane protein of the TNF ligand family that is expressed in cells such as osteoblasts and T cells (Fig. 3). RANKL-deficient mice exhibit typical osteopetrosis due to lack of osteoclasts, suggesting that RANKL is an absolute requirement for osteoclast development (28).

Anderson $e t$ al cloned a new member of the TNF receptor family, termed 'RANK', from a cDNA library of human dendritic cells. The mouse homolog was also isolated from the fetal mouse liver cDNA library (29). RANK is a transmembrane heterotrimer on the surface of hematopoietic osteoclast progenitors, mature osteoclasts, dendritic cells, and mammary gland epithelial cells (Fig. 3). It fails to bind other members of the TNF ligand family, such as Fas ligand, CD40 ligand, TNF $\alpha$ or TRAIL (19). A soluble form of RANK prevents RANKL-induced osteoclastogenesis (30). RANK-deficient mice have a complete block in osteoclast development that can be rescued by transplantation of bone marrow cells from wild-type mice, indicating that they have an intrinsic defect in osteoclast function (31).

RANK, RANKL and OPG are involved in not only physiological but also pathological conditions of bone metabolism. Bone resorption is a major pathological factor in chronic 

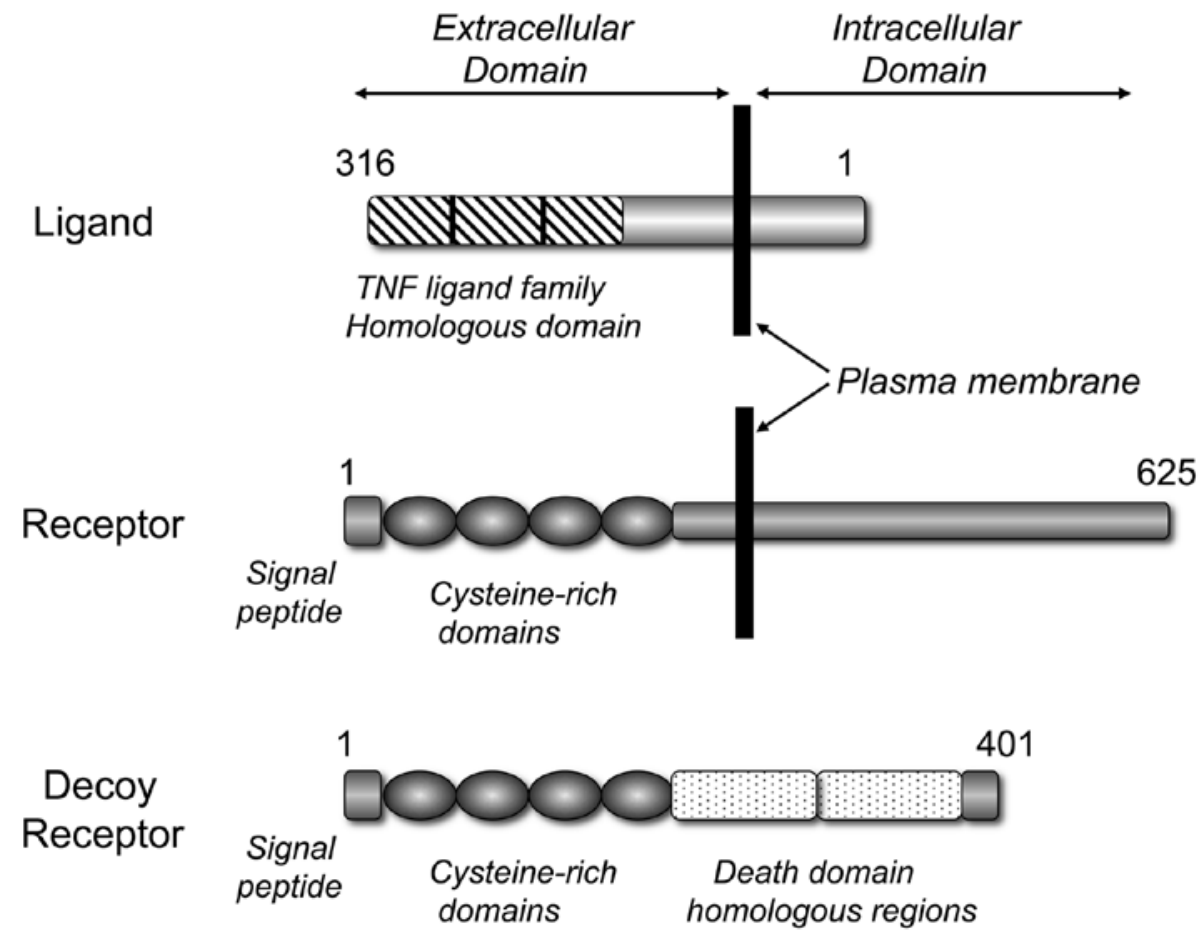

Figure 3. Molecular characteristics of RANKL, RANK and OPG. RANKL belongs to the TNF superfamily. Membrane-bound RANK and OPG belong to the TNF receptor family.

inflammatory diseases such as periodontitis, osteoporosis and arthritis. It is now clear that an imbalance in the RANKL-OPG ratio is crucial for initiating the bone loss associated with these conditions $(24,25)$. Therefore, inhibition of RANKL/RANK signaling might be effective for preventing inflammatory bone destruction. In fact, denosumab, a human monoclonal antibody against RANKL, has been shown to effectively reduce RANK signaling and thus osteoclast activity. In large, randomized, phase III studies, it has been demonstrated to prevent fractures and bone loss and improve the bone mineral density in various cancerous and non-cancerous settings (32).

\section{RANKL/RANK signaling is involved in oral SCC cell-induced osteoclastogenesis}

The dysregulation of functional equilibrium in the RANKL/RANK/OPG triad is responsible for osteolysis associated with the development of malignant tumors in bone sites. Recent studies have shown that administration of OPG or sRANK prevents bone metastasis by cancer cells in vivo (21-23). These findings suggest that the RANKL/RANK system contributes to bone metastasis by cancer cells. However, it remains controversial whether cancer cells directly express RANKL on their surface and to what extent this expression contributes to osteoclast formation.

The expression of RANKL has already been detected in several tumor cell types and can be considered a key factor in bone remodeling associated with bone metastases (33-37). Several oral SCC cells in patients and oral SCC cell lines express RANKL (38-40). Recently, Chuang et al compared RANKL expression between buccal SCC without bone invasion (25 cases) and gingival SCC with invasion (15 cases).
There were no differences between the immunohistochemical expression of RANKL in cases of buccal and gingival SCC (41). This suggests that, in cases of human buccal SCC without bone invasion, tumor cells do possess the potential to induce osteoclastogenesis through the RANKL/RANK pathway if triggered under appropriate conditions. It is possible that close proximity of the cancer to the jawbone may be a prerequisite.

It has been reported that cancer cells expressing RANKL are able to induce osteoclastogenesis even in the absence of other accessory cells $(38,42)$. On the other hand, not all cancers express RANKL, and cell-to-cell contact between cancer cells and host cells does not always lead to RANKL expression $(43,44)$. We showed in a previous study that BHY cells, which were highly invasive to the mandibular bone when inoculated into the masseter of nude mice, expressed RANKL on their cell surface but failed to induce osteoclastogenesis in cocultures of mouse bone marrow cells (BMCs) and BHY cells (39). However, adding BHY cells to a coculture of mouse POBs and BMCs markedly induced osteoclastogenesis in the absence of osteotropic factors. Consistent with these results, HSC-2 cells, which do not express RANKL, also induced osteoclast formation in cocultures of mouse BMCs and POBs without any osteotropic factors (39). The addition of BHY cells suppressed mouse OPG mRNA expression and protein production by POBs. This finding is consistent with the observation that BHY cells did not enhance osteoclastogenesis in cocultures of BMCs and POBs from OPG-deficient mice. Furthermore, immunohistochemical analysis showed a reduction of OPG expression in stromal cells from osteolytic lesions as compared to normal lesions. Therefore, oral SCC-induced suppression of OPG expression in POBs, rather than expression of RANKL in oral SCC cells, appears critical for osteoclastogenesis (Fig. 4A) (39). 

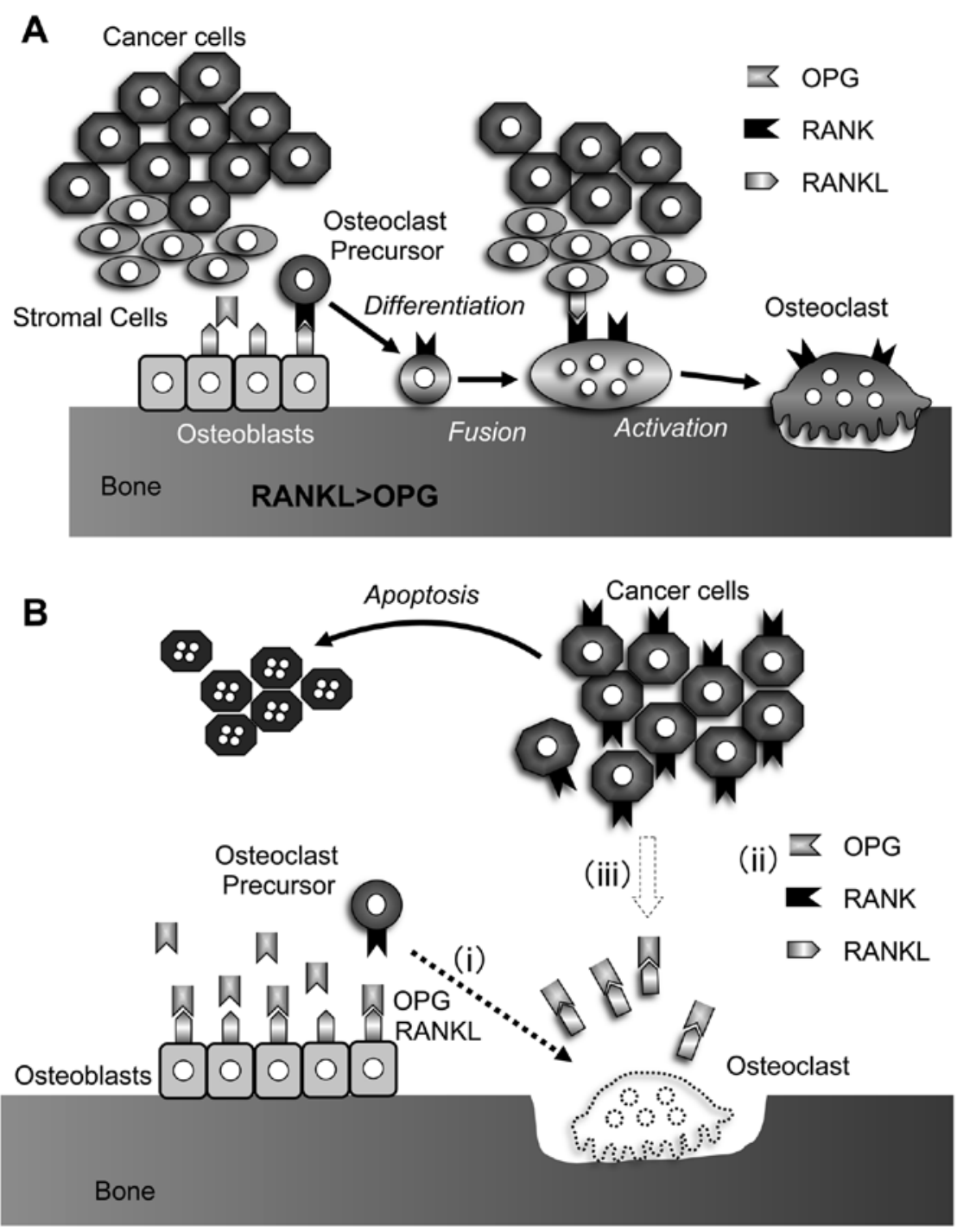

Figure 4. Possible mechanisms of OPG action on bone invasion by oral SCC. (A) Oral SCC cells induce osteoclastogenesis and bone resorption activity. (B) It is suspected that OPG suppresses mandibular bone invasion by three mechanisms; (i) OPG inhibits osteoclast differentiation; (ii) OPG also limits the supply of bone-derived growth factors, which facilitate the proliferation and survival of cancer cells that colonize bone by inhibiting osteoclastic bone resorption; (iii) furthermore, OPG suppresses RANKL-induced oral SCC cell migration.

\section{Osteoclast function regulated by RANKL/RANK signaling}

Despite the importance of oral SCC for osteoclastogenesis, its roles in osteoclast function, such as multinucleation, survival and pit-forming activity, is not fully understood. Therefore, we also examined the effect of oral SCCs on osteoclast function using BHY cells (45). Osteoclasts are terminally differentiated cells, with a short life span, that undergo rapid apoptosis in the absence of cytokines such as M-CSF, IL-1, TNF $\alpha$ or RANKL. Both BHY cells and CM from BHY cells support osteoclast survival by suppressing expression of Bim, a member of the $\mathrm{BH}$ (Bcl-2 homology) 3-only family of pro-apoptotic proteins (45). Soluble factors, such as IL- $1 \beta$, TNF $\alpha$ or RANKL, from BHY cells might contribute to the survival of osteoclasts. M-CSF, IL-1 and RANKL induced both the survival and multinucleation of prefusion osteoclasts (46). BHY cells induced not only the survival but also the multinucleation of prefusion osteoclasts.
Although the CM from BHY cells also induced multinucleation of prefusion osteoclasts, it did so less efficiently. Furthermore, adding BHY cells, but not the CM of BHY cells, induced pitforming activity of osteoclasts. Adding OPG abrogated the activity (45). Thus, oral SCC cells regulate not only osteoclast formation but also function (Fig. 4A).

\section{The role of RANKL/RANK signaling on bone invasion by oral SCCs}

The addition of OPG dramatically suppresses oral SCC regulation of both osteoclast differentiation and function in vitro, suggesting that RANKL/RANK inhibition might be an effective therapeutic approach for inhibiting oral SCC-induced bone invasion. In support of this notion, recent studies in rodent models of breast and prostate cancer have established that inhibition of RANKL/RANK signal decreases bone lesion development and tumor growth in bone (21-23). Alternatively, 
it has been reported that functional RANK is expressed on some bone-associated tumor cells $(21-23,54)$. Indeed, the migration of RANK-positive tumor cells is induced by RANKL stimulation. Thus, these observations suggest that increased RANKL expression in the tumor-bone environment is a promoting factor for bone tumor development (Fig. 2) (21-23,47).

We injected B88 human oral SCC cells into the masseter region of nude mice to establish an animal model of oral SCC bone invasion and then determine whether OPG prevents bone invasion by oral SCC in vivo. Treatment with OPG for 3 weeks decreased bone invasion by B88 cells and reduced the number of tartrate-resistant acid phosphatase (TRAP)-positive osteoclasts. OPG decreased tumor burden and increased cell death in B88 cells, whereas B88 cell mitosis was unchanged. Thus, the suppressive effect of OPG on B88 tumor burden is attributed to increased cell death rather than inhibition of proliferation. However, OPG did not affect apoptosis and proliferation of B88 cells in vitro, suggesting that effects of OPG on apoptosis in B88 cells are restricted to the bone environment (48).

Consistent with other types of cancer, tumor tissue from oral SCC patients and oral SCC cell lines, including BHY and B88, express RANK. Chuang et al reported that RANK expression was observed in oral SCC, but not normal mucosal tissue (41). RANK might become upregulated in cancer cells. Although tumor cells were closely associated with bone in controls, tumors were farther from the bone in mice treated with OPG. To support these results, RANKL enhanced B88 cell migration in a modified chemotaxis chamber equipped with a gelatin-coated filter. This effect was inhibited by OPG (48).

Taken together, RANKL/RANK inhibition suppresses bone invasion by inhibiting osteoclastogenesis and cancer cell migration and by inducing apoptosis of cancer cells via indirect anticancer action in vivo (Fig. 4B).

\section{Conclusions and perspectives}

Bone is a good environment for the progression of bone invasion. It has been suggested that oral SCC cells release soluble factors that activate osteoclast differentiation and function directly or indirectly via osteoblasts (Fig. 2) (13). During bone destruction, osteoclasts release various growth factors, including insulinlike growth factor and transforming growth factor $\beta$ (Fig. 2). This cycle has been proposed to explain tumor development in bone. The inhibition of osteoclast differentiation and function by blocking RANKL/RANK constitutes a potentially novel approach to maintaining skeletal integrity. Indeed, blocking RANKL/RANK with sRANK or OPG successfully prevents the development of bone invasion (Fig. 4).

A phase I study testing recombinant OPG in patients with multiple myeloma- or breast carcinoma-related bone metastases is currently in progress (49). Thus far, OPG has no side-effects when administered as a single subcutaneous injection to patients (50), and it has been used subcutaneously to treat bone metastases from multiple myeloma and breast cancer cells $(23,51)$. The RANKL inhibitor denosumab, a human monoclonal antibody against human RANKL, has also developed and is tested in the clinic. Denosumab was generally well tolerated throughout clinical trials without the patients having detectable anti-denosumab antibodies (32). In the case of mandibular bone invasion, it is possible to treat the cancer with OPG locally because oral SCC cells invade from cortical bone, and the tumor and defect area are at the surface of the body. However, recent reports showed that the observed incidence of osteonecrosis of the jaw was comparable to that with bisphosphonates $(52,53)$. Therefore, it is important to carefully consider the dosage and schedule of administration to avoid any side-effects of local injection of OPG or denosumab before the realization of the clinical application.

A great deal of progress has been made in understanding the pathogenesis of oral SCC. In addition, new approaches have been developed in the fields of molecular biology, cancer genetics, and cancer biology to examine the cellular and molecular mechanisms of bone invasion by oral SCCs (54). In particular, the discovery of the RANKL/RANK/OPG triad was a breakthrough in our knowledge of osteoclast biology. The development of targeted approaches against the RANKL/RANK system will contribute to preventing bone invasion by oral SCCs.

\section{Acknowledgements}

This study was supported by a Grant-in-Aid from Kyushu Dental College Internal Grants (to E.J.), the Ministry of Education, Culture, Sports, Science and Technology of Japan (to M.S. 70549261) and a Grant-in-Aid from Kyushu Dental College Alumni Association Grants (to M.S.).

\section{References}

1. Haddad RI and Shin DM: Recent advances in head and neck cancer. N Engl J Med 359: 1143-1154, 2008.

2. Cohen EE, Haraf DJ, Kunnavakkam R, et al: Epidermal growth factor receptor inhibitor gefitinib added to chemoradiotherapy in locally advanced head and neck cancer. J Clin Oncol 28: 3336-3343, 2010

3. Jimi E, Furuta H, Matsuo K, Tominaga K, Takahashi $\mathrm{T}$ and Nakanishi O: The cellular and molecular mechanisms of bone invasion by oral squamous cell carcinoma. Oral Dis 17: 462-468, 2011.

4. Ash CS, Nason RW, Abdoh AA and Cohen MA: Prognostic implications of mandibular invasion in oral cancer. Head Neck 22: 794-798, 2000.

5. Semba I, Matsuuchi $\mathrm{H}$ and Miura Y: Histomorphometric analysis of osteoclastic resorption in bone directly invaded by gingival squamous cell carcinoma. J Oral Pathol Med 25: $429-435,1996$.

6. Totsuka Y, Usui Y, Tei K, Fukuda H, Shindo M, Iizuka T, and Amemiya A: Mandibular involvement by squamous cell carcinoma of the lower alveolus: analysis and comparative study of histologic and radiologic features. Head Neck 13: 40-50, 1991.

7. Shibahara T, Noma H, Takasaki Y and Nomura T: Repair of the inferior alveolar nerve with a forearm cutaneous nerve graft after ablative surgery of the mandible. J Oral Maxillofac Surg 58: 714-717, 2000.

8. Shah J and Lydiatt WM: Buccal mucosa, alveolus, retromolar trigone, floor of mouth, hard palate, and tongue tumors. In: Comprehensive Management of Head and Neck Tumors. Thawley SE (ed). 2nd edition. WB Saunders, Philadelphia, PA, pp686-693, 1999.

9. Carter RL, Tsao SW, Burman JF, Pittam MR, Clifford P and Shaw HJ: Patterns and mechanisms of bone invasion by squamous carcinomas of the head and neck. Am J Surg 146: 451-455, 1983.

10. Müller $\mathrm{H}$ and Slootweg PJ: Mandibular invasion by oral squamous cell carcinoma. Clinical aspects. J Craniomaxillofac Surg 18: 80-84, 1990.

11. Totsuka Y, Usui Y, Tei K, Kida M, Mizukoshi T, Notani K and Fukuda H: Results of surgical treatment for squamous carcinoma of the lower alveolus: segmental vs. marginal resection. Head Neck 13: 114-120, 1991. 
12. Wong RJ, Keel SB, Glynn RJ and Varvares MA: Histological pattern of mandibular invasion by oral squamous cell carcinoma. Laryngoscope 110: 65-72, 2000.

13. Guise TA and Mundy GR: Cancer and bone. Endocr Rev 19: 18-54, 1998.

14. Shibahara T, Nomura T, Cui NH and Noma H: A study of osteoclast-related cytokines in mandibular invasion by squamous cell carcinoma. Int J Oral Maxillofac Surg 34: 789-793, 2005.

15. Yasuda H, Shima N, Nakagawa N,etal: Osteoclast differentiation factor is a ligand for osteoprotegerin/osteoclastogenesis-inhibitory factor and is identical to TRANCE/RANKL. Proc Natl Acad Sci USA 95: 3597-3602, 1998.

16. Lacey DL, Timms E, Tan HL, et al: Osteoprotegerin ligand is a cytokine that regulates osteoclast differentiation and activation. Cell 93: 165-176, 1998.

17. Tsuda E, Goto M, Mochizuki S, Yano K, Kobayashi F, Morinaga $\mathrm{T}$ and Higashio $\mathrm{K}$ : Isolation of a novel cytokine from human fibroblasts that specifically inhibits osteoclastogenesis. Biochem Biophys Res Commun 234: 137-142, 1997.

18. Simonet WS, Lacey DL, Dunstan CR, et al: Osteoprotegerin: a novel secreted protein involved in the regulation of bone density. Cell 89: 309-319, 1997.

19. Suda T, Takahashi N, Udagawa N, Jimi E, Gillespie MT and Martin TJ: Modulation of osteoclast differentiation and function by the new members of the tumor necrosis factor receptor and ligand families. Endocr Rev 20: 345-357, 1999.

20. Lee Y, Schwarz E, Davies M, et al: Differences in the cytokine profiles associated with prostate cancer cell induced osteoblastic and osteolytic lesions in bone. J Orthop Res 21: 62-72, 2003.

21. Miller RE, Branstetter D, Armstrong A, et al: Receptor activator of NF- $\kappa \mathrm{B}$ ligand inhibition suppresses bone resorption and hypercalcemia but does not affect host immune responses to influenza infection. J Immunol 179: 266-274, 2007.

22. Armstrong AP, Miller RE, Jones JC, Zhang J, Keller ET and Dougall WC: RANKL acts directly on RANK-expressing prostate tumor cells and mediates migration and expression of tumor metastasis genes. Prostate 68: 92-104, 2008.

23. Canon JR, Roudier M, Bryant R, Morony S, Stolina M, Kostenuik PJ and Dougall WC: Inhibition of RANKL blocks skeletal tumor progression and improves survival in a mouse model of breast cancer bone metastasis. Clin Exp Metastasis 25: $119-129,2008$.

24. Wada T, Nakashima T, Hiroshi $\mathrm{N}$ and Penninger JM: RANKL-RANK signaling in osteoclastogenesis and bone disease. Trends Mol Med 12: 17-25, 2006.

25. Boyle WJ, Simonet WS and Lacey DL: Osteoclast differentiation and activation. Nature 423: 337-342, 2003.

26. Mizuno A, Amizuka N, Irie K, et al: Severe osteoporosis in mice lacking osteoclastogenesis inhibitory factor/osteoprotegerin. Biochem Biophys Res Commun 247: 610-615, 1998

27. Wong BR, Rho J, Arron J, et al: TRANCE is a novel ligand of the tumor necrosis factor receptor family that activates c-Jun N-terminal kinase in T cells. J Biol Chem 272: 25190-25194, 1997.

28. Kong YY, Yoshida H, Sarosi I, et al: OPGL is a key regulator of osteoclastogenesis, lymphocyte development and lymph-node organogenesis. Nature 397: 315-323, 1999.

29. Anderson DM, Maraskovsky E, Billingsley WL, et al: A homologue of the TNF receptor and its ligand enhance T-cel growth and dendritic-cell function. Nature 390: 175-179, 1997.

30. Jimi E, Akiyama S, Tsurukai T, et al: Osteoclast differentiation factor acts as a multifunctional regulator in murine osteoclast differentiation and function. J Immunol 163: 434-442, 1999.

31. Dougall WC, Glaccum M, Charrier K, et al: RANK is essential for osteoclast and lymph node development. Genes Dev 13: 2412-2424, 1999.

32. Body JJ, Facon T, Coleman RE, et al: A study of the biological receptor activator of nuclear factor- $\kappa \mathrm{B}$ ligand inhibitor, denosumab, in patients with multiple myeloma or bone metastases from breast cancer. Clin Cancer Res 12: 12211228, 2006.

33. Bendre M, Gaddy D, Nicholas RW and Suva LJ: Breast cancer metastasis to bone: it is not all about PTHrP. Clin Orthop Relat Res 415: S39-S45, 2003.

34. Thomas RJ, Guise TA, Yin JJ, Elliott J, Horwood NJ, Martin TJ and Gillespie MT: Breast cancer cells interact with osteoblasts to support osteoclast formation. Endocrinology 140: 4451-4458, 1999.
35. Brown JM, Corey E, Lee ZD, True LD, Yun TJ, Tondravi M and Vessella RL: Osteoprotegerin and RANK ligand expression in prostate cancer. Urology 57: 611-616, 2001.

36. Sezer O, Heider U, Zavrski I, Kühne CA and Hofbauer LC: RANK ligand and osteoprotegerin in myeloma bone disease. Blood 101: 2094-2098, 2003

37. Yaccoby S, Wezeman MJ, Henderson A, Kühne CA and Hofbauer LC: Cancer and the microenvironment: myeloma-osteoclast interactions as a model. Cancer Res 64: 2016-2023, 2004.

38. Nagai M, Kyakumoto S and Sato N: Cancer cells responsible for humoral hypercalcemia express mRNA encoding a secreted form of ODF/TRANCE that induces osteoclast formation. Biochem Biophys Res Commun 269: 532-536, 2000.

39. Tada T, Jimi E, Okamoto M, Ozeki S and Okabe K: Oral squamous cell carcinoma cells induce osteoclast differentiation by suppression of osteoprotegerin expression in osteoblasts. Int J Cancer 116: 253-262, 2005.

40. Kayamori K, Sakamoto K, Nakashima T, et al: Roles of interleukin- 6 and parathyroid hormone-related peptide in osteoclast formation associated with oral cancers: significance of interleukin- 6 synthesized by stromal cells in response to cancer cells. Am J Pathol 176: 968-980, 2010.

41. Chuang FH, Hsue SS, Wu CW and Chen YK: Immunohistochemical expression of RANKL, RANK, and OPG in human oral squamous cell carcinoma. J Oral Pathol Med 38: 753-759, 2009.

42. Farrugia AN, Atkins GJ, To LB, et al: Receptor activator of nuclear factor- $\kappa \mathrm{B}$ ligand expression by human myeloma cells mediates osteoclast formation in vitro and correlates with bone destruction in vivo. Cancer Res 63: 5438-5445, 2003.

43. Giuliani N, Bataille R, Mancini C, Lazzaretti M and Barillé S: Myeloma cells induce imbalance in the osteoprotegerin/ osteoprotegerin ligand system in the human bone marrow environment. Blood 98: 3527-3533, 2001.

44. Ohshiba T, Miyaura C, Inada M and Ito A: Role of prostaglandin E produced by osteoblasts in osteolysis due to bone metastasis. Biochem Biophys Res Commun 300: 957-964, 2003.

45. Tada T, Shin M, Fukushima H, Okabe K, Ozeki S, Okamoto M and Jimi E: Oral squamous cell carcinoma cells modulate osteoclast function by RANKL-dependent and -independent mechanisms. Cancer Lett 274: 126-131, 2009.

46. Jimi E, Nakamura I, Duong LT, Ikebe T, Takahashi $\mathrm{N}$, Rodan GA and Suda T: Interleukin 1 induces multinucleation and bone-resorbing activity of osteoclasts in the absence of osteoblasts/stromal cells. Exp Cell Res 247: 84-93, 1999.

47. Jones DH, Nakashima T, Sanchez OH, et al: Regulation of cancer cell migration and bone metastasis by RANKL. Nature 440: 692-696, 2006

48. Shin M, Matsuo $\mathrm{K}$, Tada $\mathrm{T}$, et al: The inhibition of RANKL/RANK signaling by osteoprotegerin suppresses bone invasion by oral squamous cell carcinoma cells. Carcinogenesis 32: 1634-1640, 2011

49. Body JJ, Greipp P, Coleman RE, et al: A phase I study of AMGN-0007, a recombinant osteoprotegerin construct, in patients with multiple myeloma or breast carcinoma related bone metastases. Cancer 97: 887-892, 2003.

50. Bekker PJ, Holloway D, Nakanishi A, Arrighi M, Leese PT and Dunstan CR: The effect of a single dose of osteoprotegerin in postmenopausal women. J Bone Miner Res 16: 348-360 2001

51. Roodman GD and Dougall WC: RANK ligand as a therapeutic target for bone metastases and multiple myeloma. Cancer Treatment Rev 34: 143-150, 2008.

52. Steger GG and Bartsch R: Denosumab for the treatment of bone metastases in breast cancer: evidence and opinion. Ther Adv Med Oncol 3: 233-243, 2011.

53. Kyrgidis A and Toulis KA: Denosumab-related osteonecrosis of the jaws. Osteoporos Int 22: 369-370, 2011.

54. Choi S and Myers JN: Molecular pathogenesis of oral squamous cell carcinoma: implications for therapy. J Dent Res 87: 14-32, 2008. 